\title{
Quantizations and classical non-commutative non-associative algebras
}

\author{
Hilja Lisa HURU and Valentin LYCHAGIN \\ Department of Mathematics, Tromsø University, N-9037 Tromsø, Norway \\ E-mails: Hilja.Huru@matnat.uit.no and Valentin.Lychagin@matnat.uit.no
}

\begin{abstract}
In this paper we study quantizations, associativity constraints and braidings in the monoidal category of monoid graded modules over a commutative ring. All of them can be described in terms of the cohomology of underlying monoid. The case when the monoid is a finite topology has the main interest for us. The cohomology classes which are invariant with respect to homeomorphism group produce remarkable algebraic constructions. We study in details the Sierpinski and discrete topology and show the relations with the Clifford algebras, the Cayley algebra and their quantizations. All of them are $\alpha$-associative and $\sigma$-commutative for suitable associativity constraints $\alpha$ and braidings $\sigma$.
\end{abstract}

Dedicated to Maks A. Akivis on the occasion of his 85 th birthday and 65 years of scientific activity

\section{The monoidal category of graded modules}

Throughout this paper, let $M$ be a finite commutative monoid. Let $k$ be a commutative ring with unit. Denote by $k_{M}$-mod the strict monoidal category of $M$-graded $k$-modules. The objects in the category are $M$-graded $k$-modules and the arrows are the $M$-graded morphisms. $k_{M}$-mod has the strict monoidal structure where the tensor product of two objects $X=\oplus_{i \in M} X_{i}$ and $X^{\prime}=\oplus_{j \in M} X_{j}^{\prime}$ is the $k$-module $X \otimes_{k} X^{\prime}$ with grading

$$
\left(X \otimes_{k} X^{\prime}\right)_{m}=\oplus_{i+j=m}\left(X_{i} \otimes_{k} X_{j}^{\prime}\right)
$$

The ring $k$ is a unit object $e$ as we define $k$ to be indexed by $0 \in M$ and components indexed

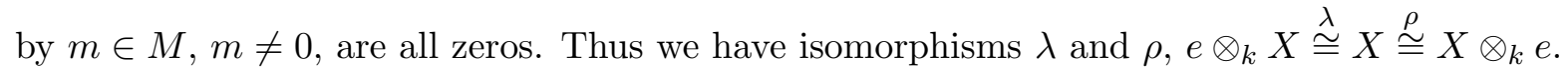

An algebra $\langle A, \mu, \eta\rangle$ in the monoidal category $k_{M}$-mod is called $M$-graded $k$-algebra. Here $\mu$ and $\eta$ are morphisms of multiplication $\mu: A \otimes A \rightarrow A$ and unit $\eta: e \rightarrow A$ such that $\mu$ is associative and unit-preserving. Note that $\mu$ maps $A_{i} \otimes A_{j}$ to $A_{i+j}$. In the same manner one defines an $M$-graded $A$-module as a module $X$ with an action $\nu: A \otimes X \rightarrow X$ in the monoidal category $k_{M}$-mod.

Let $X$ be a $M$-graded $k$-module. Denote by $\left\{\pi_{m}: X \rightarrow X\right\}_{m \in M}$ a family of projectors where $\pi_{m}$ is the projector $X$ on $X_{m}, X_{m}=\operatorname{Im}\left(\pi_{m}\right)$,

$$
\sum_{m \in M} \pi_{m}=\mathbf{1}_{X} \quad \text { and } \quad \pi_{m} \pi_{m^{\prime}}=0
$$

if $m \neq m^{\prime}$. Any such family of projectors determines a $M$-grading on a $k$-module $X$.

Consider the algebra of all $k$-valued functions on $M, k(M)$. Then functions $\left\{\theta_{m}\right\}_{m \in M}$,

$$
\theta_{m}\left(m^{\prime}\right)= \begin{cases}0 & \text { if } m^{\prime} \neq m \\ 1 & \text { if } m^{\prime}=m\end{cases}
$$


for $m, m^{\prime} \in M$, constitute a basis of the algebra. We define a $k(M)$-module structure on the $M$-graded $k$-module $X$ by putting $\theta_{m} \cdot x=\pi_{m}(x)$ for $m \in M$ and $x \in X_{m}$. For any function $f \in k(M)$ we define the action of $f$ on $X$ as follows

$$
f \cdot x=\left(\sum_{m \in M} f(m) \theta_{m}\right) \cdot x \stackrel{\text { def }}{=} \sum_{m \in M} f(m) \pi_{m}(x)
$$

If $X$ is a $k$-module, then $X$ is a $k(M)$-module if and only if it is a $M$-graded $k$-module. Hence, we get an isomorphism between the monoidal category of $M$-graded $k$-modules and the category of $k(M)$-modules.

\subsection{Group gradings and actions}

Let $G$ be a finite abelian group and $\hat{G}$ be the dual group of $G$ consisting of all group homomorphisms $G \rightarrow \mathbb{T}, \hat{G}=\operatorname{Hom}(G, \mathbb{T})$, where $\mathbb{T}=\{z \in \mathbb{C}|| z \mid=1\}$ is the 1-dimensional torus.

In this section we will show that $\hat{G}$-grading gives $G$-action, and the other way around, and that we have an isomorphism between the $\hat{G}$-graded $\mathbb{C}$-modules and $G$-modules. This isomorphism will be useful in applications of the theory developed in this paper.

Denote by $\left\{\delta_{g}\right\}_{g \in G}=\left\{\theta_{g}^{*}\right\}_{g \in G}$ the the basis consisting of Dirac $\delta$-functions in the dual of the function algebra $\mathbb{C}[G]=\mathbb{C}(G)^{*}=\operatorname{Hom}_{\mathbb{C}}(\mathbb{C}(G), \mathbb{C})$, where $\mathbb{C}[G]$ is the group algebra of $G$. Note that $G$-module structure and $\mathbb{C}[G]$-module structure induce each other.

The Fourier transform $F$ is the algebra isomorphism between $\mathbb{C}[G]=\mathbb{C}(G)^{*}$ and $\mathbb{C}(\hat{G})$

$$
F: \mathbb{C}[G] \rightarrow \mathbb{C}(\hat{G})
$$

given by $F\left(\delta_{g}\right)(\varkappa)=\varkappa(g)$, for $g \in G, \varkappa \in \hat{G}$, and

$$
F^{-1}(f)=\frac{1}{|G|} \sum_{\substack{\varkappa \in \hat{G} \\ g \in G}} \lambda_{\varkappa} \varkappa\left(g^{-1}\right) \cdot \delta_{g}
$$

where $f=\sum_{\varkappa \in \hat{G}} \lambda_{\varkappa} \theta_{\varkappa} \in \mathbb{C}(\hat{G})$. We get the following commutative diagram:

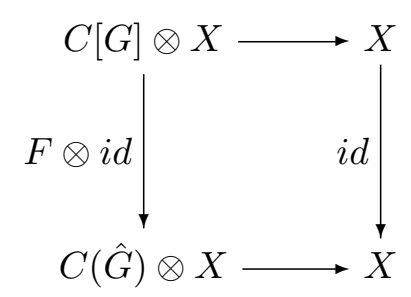

which shows that if $X$ is a $G$-module we get a $\mathbb{C}(\hat{G})$-module structure on $X$ by $f \cdot x=F^{-1}(f) \cdot x$, for $x \in X, f \in \mathbb{C}(\hat{G})$, and, conversely, if $X$ is a $\hat{G}$-graded $\mathbb{C}$-module, then we get an action of $G$ on $X$ by $g \cdot x=F\left(\delta_{g}\right) \cdot x$, for $x \in X, g \in G$. This establishes an isomorphism between the monoidal category of $G$-modules and $\hat{G}$-graded modules over $\mathbb{C}$, [3].

\section{Associativity constraints, braidings and quantizations}

Recall that an associativity constraint $\alpha$, see [7], in a monoidal category $C$ is a natural isomorphism

$$
\alpha: X \otimes(Y \otimes Z) \stackrel{\sim}{\rightarrow}(X \otimes Y) \otimes Z, \quad X, Y, Z \in O b(C)
$$


that satisfies the Mac Lane coherence condition.

A braiding of a monoidal category $C$ is a natural isomorphism

$$
\sigma=\sigma_{X, Y}: X \otimes Y \tilde{\sim} Y \otimes X
$$

for $X, Y \in O b(C)$, which preserves the unit and the associativity constraint, $\alpha \circ \sigma \circ \alpha=$ $\sigma \otimes 1 \circ \alpha \circ 1 \otimes \sigma$ and $\alpha^{-1} \circ(\sigma \otimes 1) \circ \alpha=1 \otimes \sigma \circ \alpha^{-1} \circ \sigma$. Let $A \in O b(C)$ be an algebra with multiplication $\mu: A \otimes A \rightarrow A$. We say that $A$ is $\sigma$-commutative if $\mu=\mu \circ \sigma$, see [5].

A quantization, see [6], of a monoidal category $C$ is a natural isomorphism of the tensor bifunctor

$$
q_{X, Y}: X \otimes Y \stackrel{\sim}{\rightarrow} X \otimes Y
$$

for $X, Y \in O b(C)$, which preserves the unit and the associativity, $q \circ(q \otimes 1) \circ \alpha=(q \otimes 1) \circ q \circ \alpha$.

Any braiding $\sigma$ can be quantized as follows

$$
\sigma_{X, Y}^{q}=q_{Y, X}^{-1} \circ \sigma_{X, Y} \circ q_{X, Y}
$$

and $\sigma^{q}$ is a braiding too.

We define a quantization $A_{q}$ of an algebra $A$ given by a quantization $q$ in the category $C$ to be the same object $A$ equipped with a new multiplication

$$
\mu_{q}=\mu \circ q_{A, A}: A \otimes A \rightarrow A
$$

$A_{q}=\left(A, \mu_{q}, \eta\right)$ is an algebra, see [6]. If an algebra $A$ is $\sigma$-commutative, then $A_{q}$ is $\sigma$ commutative. If $X$ is a left $A$-module in the category with the action $\nu: A \otimes X \rightarrow X$, then by a quantization $X_{q}$ of the $A$-module $X$ we mean the same object $X$ equipped with a new action

$$
\nu_{q}=\nu \circ q_{A, X}: A \otimes X \rightarrow X
$$

$X_{q}=\left(X, \nu_{q}\right)$ is also a left $A$-module in $C$, see [6].

The following theorems give the complete description of the associativity constraints, quantizations and braidings in the category of graded modules as in [3].

Theorem 1. Any associativity constraint $\alpha$ in the category $k_{M}$-mod of $M$-graded $k$-modules has the form

$$
\alpha: x_{i} \otimes\left(y_{j} \otimes z_{l}\right) \longmapsto \alpha(i, j, l)\left(x_{i} \otimes y_{j}\right) \otimes z_{l}
$$

where $\alpha: M \times M \times M \rightarrow U(k)$ is a normalized 3-cocycle, $\alpha \in Z^{3}(M, U(k))$ with values in the group of units $U(k)$. Furthermore, the orbits of all associativity constraints under the action of natural isomorphisms of the tensor bifunctor are in one-to-one correspondence with the $3^{\text {rd }}$ cohomology group $H^{3}(M, U(k))$.

Theorem 2. Any quantization $q$ of the category of $M$-graded $k$-modules has the form

$$
q: x_{i} \otimes y_{j} \longmapsto q(i, j) x_{i} \otimes y_{j}
$$

where $q: M \times M \rightarrow U(k)$ is a normalized 2-cocycle. Moreover, the orbits of all quantizations under the action of unit-preserving natural isomorphisms of the identity functor are in one-toone correspondence with the $2^{\text {nd }}$ cohomology group, $H^{2}(M, U(k))$. 
Theorem 3. A braiding $\sigma$ in the category $k_{M}$-mod a normalized 2-cochain of $M$ such that $\sigma(i, l) \sigma(l, i)=1$, that is, any braiding in $k_{M}$-mod is a symmetry. Furthermore, the following equations are satisfied

$$
\begin{aligned}
& \sigma(i+j, l)=\frac{\alpha(i, l, j)}{\alpha(i, j, l) \alpha(l, i, j)} \sigma(i, l) \sigma(j, l) \\
& \sigma(i, j+l)=\frac{\alpha(i, j, l) \alpha(j, l, i)}{\alpha(j, i, l)} \sigma(i, j) \sigma(i, l)
\end{aligned}
$$

If the associativity constraint $\alpha$ is trivial we get the bihomomorphism conditions

$$
\sigma(i+j, l)=\sigma(i, l) \sigma(j, l), \sigma(i, j+l)=\sigma(i, j) \sigma(i, l)
$$

\section{Cohomology of the Sierpinski topology}

In this section we calculate the zero, first, second and third cohomology groups of the Sierpinski topology. We will use these groups to find possible quantizations and associativity constraints of the category of modules graded by the Sierpinski topology.

The Sierpinski topology $\tau$ is the topology of the Sierpinski set; the two-point set $\Omega=\{a, b\}$ where the point $a$ is open and the point $b$ is closed. Hence, the topology $\tau$ consists of $\{\emptyset,\{a\}, \Omega\}$. Denote $\emptyset$ by $0,\{a\}$ by $a$ and $\Omega$ by 1 , then the monoid structure on $\tau$ is given by

$$
0+a=a+0=a, \quad 1+t=t+1=1 \quad \text { and } \quad a+a=a, \quad t=a, 0
$$

Note that, a $\tau$-graded $k$-algebra can be viewed as algebra

$$
A=A_{0} \oplus A_{a} \oplus A_{1}
$$

where $A_{0}$ is a $k$-algebra, $A_{a}$ and $A_{1}$ are $A_{0}$-algebras and, in addition, $A_{1}$ is an $A_{a}$-algebra.

Straight forward calculations, see [2], show that cohomology groups of the Sierpinski topology with coefficients in an abelian group $G$ are the following:

$$
H^{0}(\tau, G)=G, \quad H^{1}(\tau, G)=1, \quad H^{2}(\tau, G)=1, \quad H^{3}(\tau, G)=G
$$

Hence, for the category of $\tau$-graded $k$-modules there are no non-trivial quantizations, but there are non-trivial associativity constraints. They have the following description.

Theorem 4. Any 3-cocycle $\alpha: \tau \times \tau \times \tau \rightarrow U(k)$, on the Sierpinski topology $\tau$ has the following form:

$$
\alpha(i, j, l)=r^{f_{1}(i, j)} r^{f_{2}(j, l)}
$$

where $r \in U(k), i, j, l \in \tau$, and $f_{1}, f_{2}: \tau \times \tau \rightarrow \tau$, are functions such that $f_{1}(a, 1)=f_{2}(1, a)=1$ and $f_{1}(i, j)=f_{2}(i, j)=0$ for all other $(i, j) \in \tau \times \tau$.

\section{The power algebra $\mathfrak{P}(\Omega)$}

Let $\Omega$ be a set consisting of $n$ elements $\Omega=\left\{a_{1}, \ldots, a_{n}\right\}$ and let $\mathfrak{P}(\Omega)$ denote the power algebra of $\Omega$, that is, the algebra of all subsets of $\Omega$. $\mathfrak{P}(\Omega)$ has the group structure with respect to symmetric difference $(A, B) \longmapsto A \square B=A \cup B \backslash A \cap B$. The passing to characteristic functions gives us an isomorphism from this group to the additive group of characteristic functions on $\Omega$ with values in $\mathbb{Z}_{2}=\{0,1\}$, hence $\mathfrak{P}(\Omega) \cong\left(\mathbb{Z}_{2}\right)^{n}$.

From now on we distinguish the following two group structures; the multiplicative group, $\left(\mathbb{Z}_{2}, \cdot\right)=(\{1,-1\}, \cdot)$, denoted by $\mathbb{Z}_{2}$, and the additive group, $\left(\mathbb{Z}_{2},+\right)=(\{0,1\},+)$, denoted by $\mathbb{Z}_{2}^{+}$. 


\subsection{The Fourier-Hadamard transform}

Let $k \supset \mathbb{Q}$ and $X$ be a $k$-module and $\rho: \mathbb{Z}_{2} \rightarrow A u t_{\mathbb{R}}(X)$ be a representation. Note that $\rho(-1)^{2}=\rho(1)=1$. Then the operators

$$
\pi_{1}=\frac{(\rho(1)-\rho(-1))}{2} \quad \text { and } \quad \pi_{2}=\mathbf{1}-\pi_{1}=\frac{(\rho(1)+\rho(-1))}{2}
$$

are projectors such that $X=\pi_{1}(X) \oplus \pi_{2}(X)$ is a $\mathbb{Z}_{2}$-graded $k$-module.

Let $\left\{\theta_{1}, \theta_{-1}\right\}$ be the basis of $k\left(\mathbb{Z}_{2}^{*}\right)$ as a vector space over $k$ and let $\left\{\delta_{1}, \delta_{-1}\right\}=\left\{\theta_{1}^{*}, \theta_{-1}^{*}\right\}$ the basis of $k\left[\mathbb{Z}_{2}\right]=k\left(\mathbb{Z}_{2}\right)^{*}$. We have

$$
\delta_{m} \cdot X=\rho(m)(X)
$$

for all $m \in \mathbb{Z}_{2}$. If we define

$$
\theta_{1} \cdot X=\pi_{2}(X)=\frac{\left(\delta_{1}+\delta_{-1}\right)}{2} \cdot X \quad \text { and } \quad \theta_{-1} \cdot X=\pi_{1}(X)=\frac{\left(\delta_{1}-\delta_{-1}\right)}{2} \cdot X
$$

then we get $k\left(\mathbb{Z}_{2}\right)$-module structure on $X$. This operator one can consider as a "change of rings", see [3], with respect to the algebra isomorphism

$$
F: k\left(\mathbb{Z}_{2}\right) \rightarrow k\left[\mathbb{Z}_{2}\right]
$$

where

$$
\theta_{1} \longmapsto \frac{\left(\delta_{1}+\delta_{-1}\right)}{2} \text { and } \theta_{-1} \longmapsto \frac{\left(\delta_{1}-\delta_{-1}\right)}{2}
$$

This is the Fourier transform for $\mathbb{Z}_{2}$. For $\left(\mathbb{Z}_{i}\right)^{n}$ we define an algebra isomorphism

$$
k\left(\left(\mathbb{Z}_{i}\right)^{n}\right)=\underbrace{k\left(\mathbb{Z}_{i}\right) \otimes \cdots \otimes k\left(\mathbb{Z}_{i}\right)}_{n \text {-times }} \stackrel{F \otimes \cdots \otimes F}{\simeq} k\left[\left(\mathbb{Z}_{2}\right)^{n}\right]=\underbrace{k\left[\mathbb{Z}_{i}\right] \otimes \cdots \otimes k\left[\mathbb{Z}_{2}\right]}_{n \text {-times }}
$$

This is the Fourier-Hadamard transform and it allows us to establish an isomorphisms between the monoidal categories of $\mathbb{Z}_{2}$-graded modules and of $\mathbb{Z}_{2}$-modules.

\subsection{The cohomology group of $\mathfrak{P}(\Omega)$ with coefficients in $U(\mathbb{R})$.}

Here we describe cohomology of the power algebra $\mathfrak{P}(\Omega)$ with coefficients $U(\mathbb{R})$ in a form suitable for us. Let $k=\mathbb{R}$ and $U(k)=\mathbb{R} \backslash\{0\}$. We only need to calculate the cohomology with coefficients in $\mathbb{Z}_{2} \subset U(\mathbb{R})$. Now,

$$
H^{r}\left(\mathbb{Z}_{2}^{+}, \mathbb{Z}_{2}^{\cdot}\right)=\left(\mathbb{Z}_{2}, \cdot\right)
$$

for all $r \geq 0$. The trivial cohomology classes are represented by $f=1$. The non-trivial cohomology classes are represented by $f \in Z^{r}\left(\mathbb{Z}_{2}^{+}, \mathbb{Z}_{2}\right)$, with $f\left(x_{1}, \ldots, x_{r}\right)=(-1)^{x_{1} \cdots x_{r}}$. Hence,

$$
H^{*}\left(\mathbb{Z}_{2}, \mathbb{Z}_{2}^{*}\right) \cong \mathbb{Z}_{2}[l]
$$

where $\mathbb{Z}_{2}[l]$ is the polynomial algebra with coefficients in $\mathbb{Z}_{2}$.

Let $n \geq 2$. The correspondence $P \in C^{r}\left(M, \mathbb{Z}_{2}^{+}\right) \longmapsto(-1)^{P} \in C^{r}\left(M, \mathbb{Z}_{2}\right)$ establishes an isomorphism between $H^{r}\left(M, \mathbb{Z}_{2}^{+}\right)$and $H^{r}\left(M, \mathbb{Z}_{2}^{i}\right)$ for any monoid $M$. Therefore

$$
H^{*}\left(\left(\mathbb{Z}_{2}^{+}\right)^{n}, \mathbb{Z}_{2}\right) \cong H^{*}\left(\left(\mathbb{Z}_{2}^{+}\right)^{n}, \mathbb{Z}_{2}^{+}\right) \cong \underbrace{H^{*}\left(\mathbb{Z}_{2}^{+}, \mathbb{Z}_{2}^{+}\right) \otimes \ldots \otimes H^{*}\left(\mathbb{Z}_{2}^{+}, \mathbb{Z}_{2}^{+}\right)}_{n \text {-times }}
$$


by the Künneth formula, we get

$$
H^{*}\left(\left(\mathbb{Z}_{2}^{+}\right)^{n}, \mathbb{Z}_{2}^{*}\right) \cong \mathbb{Z}_{2}\left[l_{1}\right] \otimes \ldots \otimes \mathbb{Z}_{2}\left[l_{n}\right] \cong \mathbb{Z}_{2}\left[l_{1}, \ldots, l_{n}\right]
$$

where each $l_{i}$ is the $i^{\text {th }}$ coordinate function from $\left(\mathbb{Z}_{2},+\right)^{n}$ to the commutative ring $\mathbb{Z}_{2}$. Hence, for the power algebra we get

$$
H^{r}\left(\mathfrak{P}(\Omega), \mathbb{Z}_{2}^{*}\right) \cong \mathbb{Z}_{2}^{r}\left[l_{1}, \ldots, l_{n}\right]
$$

which is the subspace of the polynomial algebra consisting of all homogeneous polynomials of degree $r$ with coefficients in $\left(\mathbb{Z}_{2},+, \cdot\right)$. Each $f \in C^{r}\left(\mathfrak{P}(\Omega), \mathbb{Z}_{2}\right)$ has the form

$$
f\left(x_{1}, \ldots, x_{r}\right)=(-1)^{P\left(l_{1}, \ldots, l_{n}\right)\left(x_{1}, \ldots, x_{r}\right)}
$$

where

$$
P\left(l_{1}, \ldots, l_{n}\right)\left(x_{1}, \ldots, x_{r}\right)=\sum_{i_{1}, \ldots i_{r}=1}^{n} a_{i_{1} \ldots i_{r}} l_{i_{1}}\left(x_{1}\right) \cdots l_{i_{r}}\left(x_{r}\right)
$$

is a homogeneous polynomial of degree $r$ and $a_{i_{1} \ldots i_{r}} \in \mathbb{Z}_{2}^{+}$. It follows that a representative $f$ for the cohomology classes of degree 1 is represented by a vector in the vector space $\left(\mathbb{Z}_{2}\right)^{n}$ a representative of the cohomology of degree 2 is represented by a symmetric $n \times n$ matrix $A=\left(a_{i j}\right)$ with entries in $\mathbb{Z}_{2}^{+}$, and similarly for higher dimensions.

\section{$5 \quad$ Aut $(\Omega)$-invariant cohomology groups}

In this section we investigate quantizations and associativity constraints on $\mathfrak{P}(\Omega)$-graded algebras invariant under permutations.

Denote by $A u t(\Omega) \cong S_{n}$ the group of all automorphisms of $\Omega$. Any $s \in$ Aut $(\Omega)$ introduces an automorphism on the power algebra $\mathfrak{P}(\Omega)$ by

$$
B \mapsto \chi_{B} \circ s^{-1}
$$

$B \in \mathfrak{P}(\Omega)$ and $\chi_{B}$ is the characteristic function of $B$ on $\Omega$. We need cohomologies represented by Aut $(\Omega)$-invariant cohomology classes, i.e. that are independent on the labelling of the elements of $\Omega$. They correspond to $f \in Z^{r}\left(\mathfrak{P}(\Omega), \mathbb{Z}_{2}\right), f=(-1)^{P}, P \in Z^{r}\left(\mathfrak{P}(\Omega), \mathbb{Z}_{2}^{+}\right)$where if $P$ is symmetric and therefore is a polynomial of the elementary symmetric polynomials $s_{1}, \ldots, s_{n}$ in $n$ variables $l_{1}, \ldots, l_{n}$.

\subsection{The Aut $(\Omega)$-invariant second cohomology and quantizations}

Let a representative of a non-trivial second cohomology class $f=(-1)^{P} \in Z^{2}\left(\mathfrak{P}(\Omega), \mathbb{Z}_{2}\right)$ be Aut $(\Omega)$-invariant. Then $P$ is a $\mathbb{Z}_{2}$-linear combination of the symmetric polynomials

$$
P \in\left\{s_{1}^{2}, s_{2}, s_{1}^{2}+s_{2}\right\}
$$

\subsubsection{Quantizations of the two-point algebra}

For the case $n=1, \Omega$ has only one point, $\mathfrak{P}(\Omega) \simeq\left(\mathbb{Z}_{2},+\right)$ and we have only one symmetric quantization, $q=(-1)^{s_{1}^{2}}$. 
Consider invariant quantizations of the $\mathbb{Z}_{2}^{+}$-graded algebra $A=A_{\overline{0}} \oplus A_{\overline{1}}=\mathbb{R}\left[\mathbb{Z}_{2}^{+}\right]$(the twopoint algebra) with the basis $\{1, e\}$, with the property $e^{2}=1$. We get the quantized $\mathbb{R}$-algebra $A_{s_{1}^{2}}$ by introducing a new multiplication, ${ }_{s_{1}^{2}}$, on $A$. For $e \in A_{\overline{1}}$,

$$
e *_{s_{1}^{2}} e=(-1)^{s_{1}^{2}} e^{2}=(-1)^{l(1) l(1)}=-1
$$

$A_{s_{1}^{2}}$ has as the basis $\{1, e\}$ with the property $e^{2}=-1$, hence $A_{s_{1}^{2}}=\mathbb{C}$. If we now again quantize $A_{s_{1}^{2}}$ by $q$ we are back at the monoidal algebra $\mathbb{R}\left[\mathbb{Z}_{2}^{+}\right]$.

\subsubsection{Quantizations of the four-point algebra}

For $n \geq 2$ we have the three non-trivial possibilities for $P$ described above. For the case $n=2$, $\Omega$ consists of two points and $\mathfrak{P}(\Omega)$ has four elements.

Let $A=\mathbb{R}\left[\left(\mathbb{Z}_{2}^{+}\right)^{2}\right]$ be the four-point algebra of rank 4 over $\mathbb{R}$ with the basis $\left\{1, e_{1}, e_{2}, e_{1} e_{2}\right\}$ where $e_{1}$ corresponds to the grading by $(1,0)$ and $e_{2}$ to $(0,1)$ and has the properties $e_{1}^{2}=1=$ $e_{2}^{2}$ and $e_{1} e_{2}=e_{2} e_{1}$. We get the following quantizations of this algebra.

The matrix algebra, $\operatorname{Mat}_{2}(\mathbb{R}):$ Take $P=s_{2}=l_{1} l_{2}$. Then the quantized algebra $A_{s_{2}}$ is the algebra with properties

$$
\begin{aligned}
e_{1} *_{s_{2}} e_{1} & =1 \\
e_{2} *_{s_{2}} e_{2} & =1 \\
e_{1} *_{s_{2}} e_{2}+e_{2} *_{s_{2}} e_{1} & =0
\end{aligned}
$$

This algebra is isomorphic to $\mathrm{Mat}_{2}(\mathbb{R})$.

The tensor algebra, $\mathbb{C} \otimes_{\mathbb{R}} \mathbb{C}: \quad$ Now take $P=s_{1}^{2}=l_{1}^{2}+l_{2}^{2}$. Then for the quantized algebra $A_{s_{1}^{2}}$ is the algebra with properties

$$
\begin{aligned}
& e_{1} *_{s_{1}^{2}} e_{1}=-1 \\
& e_{2} *_{s_{1}^{2}} e_{2}=-1 \\
& e_{1} *_{s_{1}^{2}} e_{2}=e_{2} *_{s_{1}^{2}} e_{1}
\end{aligned}
$$

which is isomorphic to $\mathbb{C} \otimes_{\mathbb{R}} \mathbb{C}$.

Quaternions, $\mathbb{H}$ : Take $P=s_{1}^{2}+s_{2}=l_{1} l_{2}+l_{1}^{2}+l_{2}^{2}$. Then in the quantized algebra $A_{s_{1}^{2}+s_{2}}$ is the algebra with properties

$$
\begin{aligned}
e_{1} * s_{1}^{2}+s_{2} & e_{1}=-1 \\
e_{2} * s_{1}^{2}+s_{2} & e_{2}=-1 \\
e_{1} *_{s_{1}^{2}+s_{2}} e_{2}+e_{2} *_{s_{1}^{2}+s_{2}} e_{1} & =0
\end{aligned}
$$

which is isomorphic to the quaternion algebra $\mathbb{H}$.

Remark 1. The 3 algebras we now have described, $\mathbb{C} \otimes_{\mathbb{R}} \mathbb{C}, M_{2}(\mathbb{R}), \mathbb{H}$, make out the complete list of the semisimple $\mathbb{R}$-algebras of rank 4 , and the two last ones are the Clifford algebras. 


\subsubsection{Quantization of the $n$-point algebra}

For the case $n \geq 2$ we get 3 different quantizations of the $n$-point algebra $A^{n}=\mathbb{R}\left[\left(\mathbb{Z}_{2}^{+}\right)^{n}\right]$. For the cohomology class represented by $P=s_{2}$ the quantized algebra $A_{s_{2}}^{n}$ has a basis consisting of all combinations $e_{i_{1}} * \cdots * e_{i_{r}}$ with $i_{1}<\cdots<i_{r}, 1 \leq r \leq n$ satisfying

$$
\begin{aligned}
& e_{i} *_{s_{2}} e_{i}=1, \quad i=1, \ldots, n \\
& e_{i} *_{s_{2}} e_{j}+e_{j} *_{s_{2}} e_{i}=0, \quad i, j=1, \ldots, n
\end{aligned}
$$

$A_{s_{2}}^{n}$ is isomorphic to the Clifford algebra $C_{n}^{\prime}$.

For $P=s_{1}^{2}$ the algebra $A_{s_{1}^{2}}^{n}$ has the same basis but with the properties

$$
\begin{aligned}
e_{i} *_{s_{1}^{2}} e_{i} & =-1, \quad i=1, \ldots, n \\
e_{i} *_{s_{1}^{2}} e_{j} & =e_{j} *_{s_{1}^{2}} e_{i}, \quad i, j=1, \ldots, n
\end{aligned}
$$

$A_{s_{1}^{2}}^{n}$ is isomorphic to $\underbrace{\mathbb{C} \otimes_{\mathbb{R}} \cdots \otimes_{\mathbb{R}} \mathbb{C}}_{n \text {-times }}$.

Further, for $P=s_{2}+s_{1}^{2}$ we get the algebra $A_{s_{2}+s_{1}^{2}}^{n}$ with the basis as above with the properties

$$
\begin{aligned}
e_{i} *_{s_{2}+s_{1}^{2}} e_{i} & =-1, \quad i=1, \ldots, n \\
e_{i} *_{s_{2}+s_{1}^{2}} e_{j}+e_{j} *_{s_{2}+s_{1}^{2}} e_{i} & =0, \quad i, j=1, \ldots, n
\end{aligned}
$$

$A_{s_{2}+s_{1}^{2}}^{n}$ is isomorphic to the Clifford algebra $C_{n}$.

\subsection{The Aut $(\Omega)$-invariant third cohomology and associativity constraints}

For $\Omega$ with $n$ elements let $\alpha=(-1)^{P} \in Z^{3}\left(\mathfrak{P}(\Omega), \mathbb{Z}_{2}\right)$ be an Aut $(\Omega)$-invariant representation of a third cohomology class of $\mathfrak{P}(\Omega)$ with coefficients in $\mathbb{Z}_{2}$. There are 7 possibilities for Aut $(\Omega)$ invariant $P$ :

$$
P \in\left\{s_{1}^{3}, s_{2} s_{1}, s_{3}, s_{1}^{3}+s_{2} s_{1}, s_{1}^{3}+s_{3}, s_{2} s_{1}+s_{3}, s_{1}^{3}+s_{2} s_{1}+s_{3}\right\}
$$

The representatives $\alpha$ of cohomology groups of degree 3 are associativity constraints on $\mathfrak{P}(\Omega)$ graded $\mathbb{R}$-algebras $A=\sum_{i \in \mathfrak{P}(\Omega)} A_{i}$ such that

$$
a_{i}\left(a_{j} a_{l}\right)=\alpha(i, j, l)\left(a_{i} a_{j}\right) a_{l}
$$

We require $\alpha(i, i, i)=(-1)^{P(i, i, i)}=1$, that is, $P(i, i, i)=0$.

Let $\Omega$ consist of one point. Then we have one possible symmetric associativity constraint $P=s_{1}^{3}=l^{3}$, but this does not satisfy $P(i, i, i)=0$.

Let $\Omega$ consist of two points. We have 3 possibilities for $P$, that is, $s_{1}^{3}, s_{1} s_{2}$ and $s_{1}^{3}+s_{1} s_{2}$. Only $P=s_{1} s_{2}$ does satisfy the condition $P(i, i, i)=0$, but $s_{1} s_{2}=0$ as a function over $\left(\mathbb{Z}_{2},+\right)$, so in this case we only have the trivial associativity constraint.

\section{The Cayley algebra}

Let $\Omega$ consist of three points. For algebras graded by $\mathfrak{P}(\Omega)$ there are 7 possibilities for $P$, but only $P=s_{1} s_{2}+s_{3}$ satisfies the property $P(i, i, i)=0$. 
Let $\left\{1, e_{1}, e_{2}, e_{3}, e_{1} e_{2}, e_{1} e_{3}, e_{2} e_{3}, e_{1} e_{2} e_{3}\right\}$ be the basis of the Cayley algebra or octonions $\mathfrak{C}$, [8], with the multiplication table

\begin{tabular}{|l|l|l|l|l|l|l|l|l|}
\hline & 1 & $e_{1}$ & $e_{2}$ & $e_{1} e_{2}$ & $e_{3}$ & $e_{1} e_{3}$ & $e_{2} e_{3}$ & $e_{1} e_{2} e_{3}$ \\
\hline 1 & 1 & $e_{1}$ & $e_{2}$ & $e_{1} e_{2}$ & $e_{3}$ & $e_{1} e_{3}$ & $e_{2} e_{3}$ & $e_{1} e_{2} e_{3}$ \\
\hline$e_{1}$ & $e_{1}$ & -1 & $-e_{1} e_{2}$ & $e_{2}$ & $-e_{1} e_{3}$ & $e_{3}$ & $e_{1} e_{2} e_{3}$ & $-e_{2} e_{3}$ \\
\hline$e_{2}$ & $e_{2}$ & $e_{1} e_{2}$ & -1 & $-e_{1}$ & $-e_{2} e_{3}$ & $-e_{1} e_{2} e_{3}$ & $e_{3}$ & $e_{1} e_{3}$ \\
\hline$e_{1} e_{2}$ & $e_{1} e_{2}$ & $-e_{2}$ & $e_{1}$ & -1 & $-e_{1} e_{2} e_{3}$ & $e_{2} e_{3}$ & $-e_{1} e_{3}$ & $e_{3}$ \\
\hline$e_{3}$ & $e_{3}$ & $e_{1} e_{3}$ & $e_{2} e_{3}$ & $e_{1} e_{2} e_{3}$ & -1 & $-e_{1}$ & $-e_{2}$ & $-e_{1} e_{2}$ \\
\hline$e_{1} e_{3}$ & $e_{1} e_{3}$ & $-e_{3}$ & $e_{1} e_{2} e_{3}$ & $-e_{2} e_{3}$ & $e_{1}$ & -1 & $e_{1} e_{2}$ & $-e_{2}$ \\
\hline$e_{2} e_{3}$ & $e_{2} e_{3}$ & $-e_{1} e_{2} e_{3}$ & $-e_{3}$ & $e_{1} e_{3}$ & $e_{2}$ & $-e_{1} e_{2}$ & -1 & $e_{1}$ \\
\hline$e_{1} e_{2} e_{3}$ & $e_{1} e_{2} e_{3}$ & $e_{2} e_{3}$ & $-e_{1} e_{3}$ & $-e_{3}$ & $e_{1} e_{2}$ & $e_{2}$ & $-e_{1}$ & -1 \\
\hline
\end{tabular}

$\mathfrak{C}$ is an alternating algebra of rank 8 over $\mathbb{R}$ and is in classical point of view nonassociative and noncommutative $\mathfrak{C}$ is obviously graded by $\mathfrak{P}(\Omega)$.

Let $1, e_{1}, e_{2}$ and $e_{3}$ have the gradings $(0,0,0),(1,0,0),(0,1,0)$ and $(0,0,1)$ respectively in $\left(\mathbb{Z}_{2}^{+}\right)^{3}$. We see that the Cayley algebra is $\alpha$-associative with respect to the associativity constraint

$$
\alpha=(-1)^{s_{1} s_{2}+s_{3}}
$$

and is $\sigma$-commutative with respect to the symmetry

$$
\sigma=(-1)^{l_{1} l_{2}+l_{2} l_{1}+l_{1} l_{3}+l_{3} l_{1}+l_{2} l_{3}+l_{3} l_{2}+\delta(g)}
$$

where $g((1,1,1))=1$ and $g(x)=0$ for $x \neq(1,1,1)$.

If we now quantize $\mathfrak{C}$, the three non-trivial $A u t(\Omega)$-invariant quantizations, $q_{1}=(-1)^{s_{2}}$, $q_{2}=(-1)^{s_{1}^{2}}$ and $q_{3}=(-1)^{s_{1}^{2}+s_{2}}$, the result is new $\alpha$-associative algebras with commutativities different from the commutativity of $\mathfrak{C}$. Denote these by $\mathfrak{C}_{s_{2}}, \mathfrak{C}_{s_{1}^{2}}$ and $\mathfrak{C}_{s_{1}^{2}+s_{2}}$ respectively. All are $\alpha$-associative as quantizations preserve the associativity given by $\alpha$. They have the basis of $1, e_{1}, e_{2}, e_{3}$ and all combinations $e_{i_{1}} \cdots e_{i_{r}}$ with $i_{1}<\cdots<i_{r}, 1 \leq r \leq 3$ satisfying

$$
e_{i}\left(e_{j} e_{l}\right)=-\left(e_{i} e_{j}\right) e_{l}, \quad i, j, l=1,2,3
$$

For $\mathfrak{C}_{s_{2}}$ the basis has the properties

$$
e_{i}^{2}=-1, e_{i} e_{j}=e_{j} e_{i}, \quad i, j=1,2,3
$$

Further, the properties of $\mathfrak{C}_{s_{1}^{2}}$ are

$$
e_{i}^{2}=1, e_{i} e_{j}=-e_{j} e_{i}, \quad i, j=1,2,3
$$

$\mathfrak{C}_{s_{1}^{2}+s_{2}}$ has the properties

$$
e_{i}^{2}=1, e_{i} e_{j}=e_{j} e_{i}, \quad i, j=1,2,3
$$

Assume $\Omega$ is of dimension $n$. The $2^{n}$-dimensional Cayley algebra $\mathfrak{C}_{n}$ is in the category of $\mathfrak{P}(\Omega)$ -graded modules equipped with the associativity constraint $\alpha=(-1)^{s_{1} s_{2}+s_{3}}$ and the symmetry

$$
\sigma=(-1)^{l_{1} l_{2}+l_{2} l_{1}+l_{1} l_{3}+l_{3} l_{1}+l_{2} l_{3}+l_{3} l_{2}+\delta(g)}
$$

Note that $\mathfrak{C}_{n}$ has the basis consisting of all combinations $e_{i_{1}} \cdots e_{i_{r}}$ with $i_{1}<\cdots<i_{r}, 1 \leq r \leq n$ satisfying

$$
e_{i}^{2}=-1, \quad i=2, \ldots, n
$$




$$
\begin{aligned}
e_{i} e_{j} & =-e_{j} e_{i}, \quad i, j=1, \ldots, n \\
e_{i}\left(e_{j} e_{l}\right) & =-\left(e_{i} e_{j}\right) e_{l}, \quad i, j, l=1, \ldots, n
\end{aligned}
$$

When $\mathfrak{C}_{n}$ is quantized we get the following 3 algebras with the same basis and all are $\alpha$-associative with respect to $\alpha=(-1)^{s_{1} s_{2}+s_{3}}$ such that

$$
e_{i}\left(e_{j} e_{l}\right)=-\left(e_{i} e_{j}\right) e_{l}
$$

for all $i, j, l=1, \ldots, n$. The quantization $q_{1}=(-1)^{s_{2}}$ produces the algebra $\mathfrak{C}_{n, s_{2}}$ such that

$$
\begin{aligned}
e_{i}^{2} & =-1, \quad i=2, \ldots, n \\
e_{i} e_{j} & =e_{j} e_{i}, \quad i, j=1, \ldots, n
\end{aligned}
$$

$\mathfrak{C}_{n, s_{2}}$ is $\sigma^{\prime}$-commutative with respect to the symmetry

$$
\sigma^{\prime}=(-1)^{\delta(g)}
$$

The quantization $q_{2}=(-1)^{s_{1}^{2}}$ gives the algebra $\mathfrak{C}_{n, s_{1}^{2}}$ with the properties

$$
\begin{aligned}
e_{i}^{2} & =1, \quad i=2, \ldots, n \\
e_{i} e_{j} & =-e_{j} e_{i}, \quad i, j=1, \ldots, n
\end{aligned}
$$

which is $\sigma$-commutative with respect to the symmetry

$$
\sigma=(-1)^{l_{1} l_{2}+l_{2} l_{1}+l_{1} l_{3}+l_{3} l_{1}+l_{2} l_{3}+l_{3} l_{2}+\delta(g)}
$$

Furthermore, the quantization $q_{3}=(-1)^{s_{1}^{2}+s_{2}}$ produces $\mathfrak{C}_{n, s_{1}^{2}+s_{2}}$ with the properties

$$
\begin{aligned}
e_{i}^{2} & =1, \quad i=2, \ldots, n \\
e_{i} e_{j} & =e_{j} e_{i}, \quad i, j=1, \ldots, n
\end{aligned}
$$

which is $\sigma^{\prime}$-commutative, with respect to

$$
\sigma^{\prime}=(-1)^{\delta(g)}
$$

\section{References}

[1] S. Eilenberg and S. Mac Lane. Cohomology Theory in Abstract Groups. I. Ann. of Math. 48 (1947), 51-78.

[2] H. L. Huru. Quantizations and associativity constraints of the category of graded modules. Cand. Scient. Theses in Mathematics, University of Troms $\varnothing, 2002$.

[3] H. L. Huru. Associativity constraints, braidings and quantizations of modules with grading and action. Lobachevskii J. Math. 23 (2006), 5-27; http://jm.ksu.ru/vol23/110.html.

[4] D. Husemoller. Fibre Bundles. Grad. Texts in Math. 20, Springer-Verlag, New York, 1994.

[5] V. V. Lychagin. Calculus and quantizations over Hopf algebras. Acta Appl. Math. 51 (1998), 303-352.

[6] V. Lychagin. Quantizations of differential equations. Nonlinear Analysis, 47 (2001), 2621-2632.

[7] S. Mac Lane. Categories for the Working Mathematician. Grad. Texts in Math. 5, Springer-Verlag, New York, 1998.

[8] R. D. Schafer. An Introduction to Nonassociative Algebras. Pure and Appl. Math. 22, Academic Press, New York-London, 1966. 